\title{
Civilisations
}

Revue internationale d'anthropologie et de sciences

humaines

42-2 | 1993

enQuete d'identité

\section{Construction de l'étranger dans les échanges quotidiens}

\author{
Gérard Althabe
}

\section{OpenEdition}

\section{Journals}

Édition électronique

URL : http://journals.openedition.org/civilisations/2367

DOI : 10.4000/civilisations. 2367

ISSN : 2032-0442

\section{Éditeur}

Institut de sociologie de l'Université Libre de Bruxelles

\section{Édition imprimée}

Date de publication : 1 décembre 1993

Pagination : $217-227$

ISBN : 2-87263-108-9

ISSN : 0009-8140

Référence électronique

Gérard Althabe, « Construction de l'étranger dans les échanges quotidiens », Civilisations [En ligne], 42-2 | 1993, mis en ligne le 01 décembre 1996, consulté le 07 mai 2019. URL : http://

journals.openedition.org/civilisations/2367 ; DOI : 10.4000/civilisations.2367

Ce document a été généré automatiquement le 7 mai 2019.

(c) Tous droits réservés 


\title{
Construction de l'étranger dans les échanges quotidiens
}

\author{
Gérard Althabe
}

1 Le terrain de l'analyse est constitué par ceux qui se considèrent comme les autochtones; la xénophobie est d'abord leur problème, c'est chez eux qu'il faut aller étudier et non chez ceux qu'elle prend comme cible (les allogènes). Contrairement à l'évidence de ce constat, c'est dans une perspective à la marge de ce qui est la totalité des études sur la question, c'est-à-dire les recherches sur les allogènes, leur trajectoire biographique et leur mouvement d'insertion, avec d'ailleurs la spécialisation des chercheurs selon les appartenances ethnoculturelles (les trois pays du Maghreb, les Antillais, les Asiatiques, les Africains occidentaux); que s'est créé en une dizaine d'années une recherche spécialisée en la matière (cette création est passée par des programmes initiés par les Administrations). Les autochtones ne sont introduits dans le problème que par des sondages dont les résultats indicatifs sont largement insuffisants.

2 J'ai placé la question dans le champ des échanges de la vie quotidienne, et mon objet est la production de l'étranger stigmatisé, ce mode de catégorisation qui vise à exclure, à mettre en dehors des échanges. Cette catégorisation intervient dans les échanges internes à ceux qui en sont les agents (c'est une référence qu'ils partagent) et elle structure leur rencontre avec ceux qui sont ainsi produits. Dans un deuxième moment, on peut déplacer l'investigation vers ceux qui en sont la cible et analyser les réponses qu'ils élaborent à cette pratique de catégorisation.

3 Brossons à grands traits la conjoncture. Je vais dès l'abord traiter de la corrélation qui peut être établie entre la montée de la xénophobie et une crise économique et sociale dont les effets se sont de plus en plus manifestés à partir de 1975 (cette crise d'où la France semblait sortir depuis deux ou trois années, mais avec des conséquences auxquelles je ferai allusion).

4 Les zones de périphéries urbaines dans lesquelles nous nous trouvions en 1975 ont été produites dans un puissant et continu mouvement de développement économique et social initié dans les années 30 (ce qu'on appelle avec quelque nostalgie les 30 «glorieuses »). Il s'est traduit par une urbanisation généralisée, les campagnes achèvent 
de se vider, les familles des couches subalternes et moyennes sont chassées des centresvilles et retrouvent les ruraux dans les périphéries. Cette urbanisation est inscrite et cristallisée dans les trajectoires individuelles et familiales de promotion, c'est-à-dire de l'éloignement de la pauvreté, avec ses deux composantes, la précarité matérielle et les dépendances personnelles (les conquêtes sociales).

Ce mouvement de promotion lié à l'urbanisation peut être appréhendé à travers des récits biographiques, et ceux-ci se développent sur un modèle partagé : la mise à distance de l'existence des parents (le contraste entre l'existence menée au moment de l'enquête et celle de l'enfance) et l'établissement de la continuité de la promotion familiale à travers les enfants par l'école qui est le vecteur de cet ancrage dans le futur.

6 Je ne vais pas développer cette direction (on peut la saisir dans la corrélation entre les statistiques de consommation et de revenus domestiques et les récits biographiques familiaux) ; je vais essayer de montrer comment cette promotion économique et sociale généralisée, exceptionnellement massive, se manifeste dans les échanges composant la vie quotidienne ; je vais dégager la base sur laquelle ces acteurs, agents d'une promotion sociale, organisent leurs échanges dans les immeubles, les quartiers neufs qui ont surgi en peu d'années autour de chacune des villes du pays.

7 Il me faut tempérer quelque peu cette image d'un mouvement massif de promotion sociale et matérielle, une minorité de familles reste au bord du chemin, il se constitue inévitablement une minorité de laissés pour compte que l'on retrouve dans telle rue, immeuble, ou cité. Dans ce nouveau paysage urbain on décèle une scission interne entre une grande majorité impliquée dans le mouvement de promotion et une minorité de familles maintenues en dehors de la stabilité professionnelle et dont la survie est assurée par des modalités multiples d'assistance; elles sont la cible privilégiée des différentes sortes de travailleurs sociaux. D'une manière générale, l'introduction de l'agent de l'autorité extérieure dans leur domaine privé est légitimée par la protection des enfants. D'autre part cette assistance se veut pédagogique, la situation de ces familles est considérée comme accidentelle, un retard que l'assistance comblera.

8 Ces minorités (dont l'importance quantitative est localement variable) occupent une place centrale dans les jeux de la communication que les gens mettent en oeuvre dans leur nouveau cadre de vie: d'une manière globale, ils sont produits en "acteurs symboliques » fixés à un pôle négatif, et l'axe central des échanges est la construction de la différence avec eux, l'édification de la distance envers ce pôle négatif ; c'est le cadre qui donne sens aux échanges de la vie quotidienne, ils en sont la référence centrale.

9 Je peux introduire un certain nombre de points caractérisant les modes de communication tels qu'ils se sont développés dans les territoires urbains. Nous sommes en présence d'un mode très particulier de reproduction des normes, celles-ci passant par la fixation en dehors de l'espace de communication de ceux qui cristallisent leur transgression, et la construction de la différence d'avec ces « acteurs symboliques » ainsi produits, à travers l'édification d'une frontière symbolique toujours précaire.

10 L'enfermement de ces familles dans une relation privilégiée avec les agents porteurs de l'assistance est à l'épicentre de leur production en « acteur symbolique ». On pressent que ce qu'on appelle le contrôle social exercé de l'extérieur ne passe pas par l'intervention sur une cible numériquement étroite ; à partir de la reproduction des normes, on emprunte le chemin de la construction de la distance avec ceux qui peuplent cette cible. 
11 Avec la production d'un groupe limité de jeunes en «acteurs symboliques» (ce que j'ai appelé les loubards) on retrouve la place qu'occupe la violence sociale. A travers le traitement dont ses porteurs sont l'objet se crée la frontière à l'intérieur de laquelle se constitue le champ des échanges. C'est là la tradition, dans le quotidien de ces périphéries urbaines, d'un processus fondamental que l'on retrouve à tous les niveaux de la vie sociale : à travers le refoulement à ses frontières de ceux qui sont les porteurs de la violence, c'est-à-dire des porteurs de la transgression des règles et codes, se dessine le cadre de la vie sociale interne, les échanges au champ social ainsi constitué se développent sur la base de ces règles et normes rendant possible la connivence urbaine.

La cohabitation avec les familles assistées introduit une précarité fondamentale dans la manière dont se traduit dans les échanges la promotion sociale et matérielle; elle engendre cette tension qui a son tour engendre les mille modes de construction de la frontière symbolique, d'isolement et d'extériorisation dans un monde autre de ces familles assistées.

13 Le dénouement de la tension passera par la sortie des immeubles collectifs et l'installation dans les lotissements de maisons individuelles dont on acquiert la propriété à crédit (ce mouvement est massif, il intéresse des centaines de milliers de familles). J'ai effectué des enquêtes dans ce qu'on appelle les «nouveaux villages»: le mode de communication à travers lequel, en dehors de l'espace de cohabitation, des signes pouvant marquer la présence de la couche sociale inférieure et le développement d'une sociabilité supérieure sont mis en scène ; pratiques qui ont pour acteurs des familles qui, en suivant des critères professionnels ou financiers, pourraient être classées dans les deux strates sociales.

Durant cette période de développement économique et de promotion sociale, durant la période où se met en place ce cadre urbain des échanges, ceux qu'à ce moment-là on appelle les «travailleurs immigrés » arrivent en grand nombre, mais ils restent en dehors du jeu que j'ai décrit; à l'entrée des années 70, la figure du "travailleur immigré », l'homme seul en situation de passage, est dominante. Il est destiné à repartir, l'argent qu'il vient d'épargner en France est destiné à alimenter les relations familiales constitutives de son univers social de l'origine et à y introduire une trajectoire promotionnelle par la construction d'une maison (une telle situation est actuellement celle des immigrés maliens, la migration relevant de la logique des rapports sociaux constitutifs des villages de la vallée du Sénégal).

15 A ce moment-là, les travailleurs immigrés ne sont pas implantés dans la société française, leur extériorité est marquée par leur résidence dans les bidonvilles qui sont considérés comme des fragments du Tiers Monde aux portes des agglomérations. (Ce sont des territoires étrangers, qui d'ailleurs deviendront, pour une courte période (1969-72), les terrains privilégiés des activités missionnaires des militants des groupes de l'extrême gauche nés de mai 1968.)

Quoique hors de la société française, la présence de cette population d'immigrés intervient, indirectement, dans la conjoncture qui a été décrite :

17 a) Ces immigrés maintenus dans une position d'étrangers occupent massivement les emplois subalternes, déqualifiés (la construction, les chaînes industrielles), et ces positions sont en quelque sorte extériorisées dans la mesure où elles sont occupées par les étrangers. Ils sont maintenus à la marge, quasi en dehors de la classe ouvrière, c'est là 
un domaine de tensions internes aux syndicats qui n'ont pas réussi à représenter ces couches de salariés (les femmes, les jeunes ruraux).

b) Cette installation d'immigrés dans les positions subalternes de la production joue un rôle indirect dans la production des "pauvres" autochtones comme "acteurs symboliques »: les hommes qui en font partie sont totalement en dehors du travail salarié, il n'y a pas une continuité entre la majorité et cette minorité, mais une séparation brutale, ce qui a facilité le processus décrit de production de « l'acteur symbolique ».

Tel est le paysage dans lequel intervint cette crise dont les effets vont se concrétiser dans la deuxième moitié des années 70 ; le chômage (qui va dépasser les $10 \%$ des salariés et reste d'ailleurs au même niveau actuellement) entraîne une précarisation, une fragilisation généralisées, les assurances sur lesquelles se construit la promotion sociale se décomposent, les protections sociales entourant l'emploi, qui apparaissaient comme des acquis définitifs, sont brutalement remises en cause.

Ainsi la configuration symbolique telle que je l'ai décrite est remise en question; elle a pour épicentre la construction de la différence, de la distance avec ces familles assistées et précarisées dans leur existence matérielle qui sont produites en «acteurs symboliques ». Les pratiques tendant à se protéger du refoulement vers ce pôle négatif se décomposent, perdent leur efficacité; de plus en plus de familles rejoignent cette condition de pauvreté et l'éventualité de cette chute est généralisée ; c'est le moment où l'on parle des «nouveaux pauvres ", c'est-à-dire des pauvres qui ne sont pas visibles: le pauvre est désormais mon voisin de palier!

21 Considérons l'élaboration, au niveau des échanges du quotidien où nous nous plaçons, de la réponse à la crise. Il est possible de suivre le dépassement de la production de «l'acteur symbolique »: on passe des familles assistées autochtones aux familles d'origine maghrébine. Ainsi on assiste à l'ethnicisation de la pauvreté, l'ethnicisation des porteurs de la négation des normes familiales et des porteurs de la violence (la délinquance); ainsi ce dont on se différencie est transformé en caractéristiques ethnoculturelles. C'est une manière de neutraliser dans l'imaginaire le blocage dans la promotion sociale, sa remise en question possible par le chômage, le retour à des dépendances oubliées.

22 La situation est un peu plus complexe: d'une part, on décèle la continuité, il y a continuité de la production de "l'acteur idéologique ", mais il y a extériorisation de cet acteur; d'autre part la scission indigène n'est pas dissoute, elle s'étend (de par l'accroissement des pauvres autochtones), mais elle est subordonnée au cadre précédent.

23 Ainsi l'enjeu de la cohabitation change de sens. Désormais c'est la cohabitation ethnique qui fonde la stigmatisation. Une nouvelle orientation du refus de la cohabitation est décelable; on assiste à des recompositions internes aux grands ensembles d'immeubles collectifs, avec la concentration de familles allogènes dans certains territoires urbains. L'installation dans les lotissements devient principalement la protection de la cohabitation avec les alloqués.

24 Dans la population autochtone intervient ainsi la scission entre ceux qui restent emprisonnés dans la cohabitation ethnique et ceux qui s'en sont libérés. C'est là une manière originale dont se traduit la scission constitutive de la société duale : ceux qui restent dans la cohabitation ethnique sont ceux qui partagent avec les allogènes la précarité et l'instabilité salariales. Le chômage reste désespérément constant dans son niveau quantitatif, mais il se fixe et se reproduit dans une même population. A travers la cohabitation ethnique intervient un mouvement d'extériorisation, qui bloque cette 
fraction de la population dans un univers étranger à celui d'une majorité qui lentement constitue les modes de la promotion (dans la sortie de la crise).

Je n'ai considéré la réponse à la crise que du côté des autochtones. Ce mouvement de production des étrangers (les Maghrébins comme cible privilégiée) en "acteurs symboliques » fixés au pôle négatif est corrélatif à une transformation essentielle qui se joue, dans le même moment, dans cette même population maghrébine.

Alors que jusqu'au début des années 70, la figure du travailleur immigré seul et de passage domine, on assiste durant cette décade à l'implantation d'une importante population d'origine maghrébine dans la société française.

Intervient un mouvement, favorisé par l'État, de regroupement familial (femmes et enfants rejoignent les hommes), ce qui s'accompagne de la destruction des bidonvilles (autour de 1972) et l'installation des familles dans les immeubles où elles rejoignent les autochtones. La fixation, l'insertion dans la société française passe par une tension interne à la famille, les enfants des immigrés sont scolarisés en France. Ils sont de plus en plus étrangers à leur société d'origine (ce qui se renforce d'une manière considérable avec le développement de l'Islamisme intégriste dans les pays du Maghreb). La mise à distance de la société d'origine et l'insertion dans la société française passent par la décomposition souvent dramatique, chaotique du monde familial d'origine (le père et les fils, les filles et la mère).

28 La conjoncture peut être caractérisée par la rencontre de deux processus sociaux : celui de la production des familles allogènes en "acteur symbolique » fixé au pôle négatif, réponse élaborée par les autochtones à la crise et celui, parallèle, de l'implantation d'une importante population d'origine maghrébine dans la société. Il est facile de comprendre comment le processus dont les autochtones sont les agents s'alimente de la construction d'une minorité allogène d'origine maghrébine.

L'enracinement dans la société française d'une forte minorité issue de la migration venue des trois pays du Maghreb la place devant deux modèles à travers lesquels cette présence pourra se jouer: soit ces familles empruntent le chemin de l'assimilation, soit leurs membres empruntent une voie qui finalement est l'espace public à travers leur différence ethnoculturelle, qui fournira matière à la production de leur identité collective (elle prend le plus souvent la forme religieuse islamique).

On peut désigner le drame de la situation actuelle, sa contradiction antagonique: le chemin de l'assimilation (celui emprunté par les vagues successives de migrations qui durant un siècle ont constitué la société française, et particulièrement son accomplissement avec la deuxième génération à travers l'école et les organisations politiques syndicales) est bloqué dans la mesure où le développement de la xénophobie les renvoie, les fixe dans leur différence ethnoculturelle. Ce refoulement prend une acuité particulière avec les jeunes nés de la migration. Cette catégorisation stigmatisant à travers des caractéristiques ethnoculturelles, qui transforment en étranger à une société dont on se considère membre, est d'ailleurs à l'origine d'une importante production artistique et littéraire.

31 Le chemin de l'affirmation comme acteur collectif sur la base de l'identité ethnoculturelle, ainsi que les revendications pour la construction de mosquées, d'espaces de prière dans les usines, induisent des pratiques qui renforcent et alimentent à l'infini la xénophobie, c'est-à-dire la production de cette population en étranger. C'est un cercle vicieux qui ne semble pas s'ouvrir, tout au moins pour le moment. 


\section{Une crise urbaine} et supposent l'implantation d'équipements (Centres socioculturels et Maisons de quartier ou des jeunes). Elles visent à créer des sociabilités locales et, à travers les associations, à construire ces identités collectives et leur représentation. On peut suivre sur une quinzaine d'années l'échec de ces opérations, la dissolution des associations ou leur réduction à deux ou trois personnes qui utilisent leur sigle dans la relation avec les municipalités.

3. Ce vide est le produit d'une transformation de l'intervention de la gauche politique à travers les municipalités (en 1977 les trois quarts des villes de 100.000 habitants relèvent de municipalités socialistes et communistes). Dans cette perspective, prenons une temporalité plus longue : dans les années 30 , de véritables sociétés urbaines se sont créées autour d'associations relevant de la gauche politique, chacune d'ailleurs ayant un style particulier: les communistes (avec comme centre les communes de la banlieue parisienne) ont fait du parti et des associations qui en émanaient directement le cadre dans lequel était produite cette vie sociale municipale; les socialistes (les modèles pouvant être dans le Nord autour de Lille) avec un réseau associatif directement lié à la 
municipalité, mais relativement indépendant d'un parti qui a été toujours très faible. Durant les années 60 et 70 on peut suivre le démantèlement de ces dispositifs municipaux. Ils n'ont pas réussi à maîtriser la croissance urbaine et l'arrivée d'une nouvelle population et ses recompositions successives. Autour de 1980, les militants associatifs et politiques ont disparu du paysage de ces quartiers.

Cette présentation par trop rapide désigne un lieu où la crise de la xénophobie doit se dénouer; et ce à travers des pratiques "culturelles", à l'initiative ou non des municipalités, visant à la création d'espaces locaux de communication sur la base de la production d'identités collectives locales, c'est-à-dire à la création de cadres partagés rendant possibles la négociation et la gestion de la cohabitation interethnique.

erence dans la société française d'une population allogène, placée dans une position bloquée dans la mesure où la voie de l'assimilation et celle de sa constitution en minorité ethnoculturelle reconnue lui semblent interdites, développe une crise d'une considérable ampleur. Est en jeu le modèle de société qui, jusqu'à cette décade des années 80 , a constitué un cadre idéologique accepté par tous depuis la Libération: la centralisation politique et administrative s'accompagne d'un strict partage entre la sphère du privé familial, dans laquelle ont été refoulées et sont maintenues enfermées les manifestations relevant des différences ethnoculturelles et religieuses, et un champ public centré sur un Etat qui ne connaît que des individus formellement égaux, possédant des droits politiques et sociaux relevant de la loi s'imposant à tous sans distinction. C'est en quelque sorte le champ constitutif de la société engendrée dans le territoire, et dans lequel vont se dérouler affrontements politiques et luttes sociales.

41 Ce modèle est puissamment assimilateur. Tous ceux qui sont implantés dans la société française sont pris dans la production de ce partage entre un privé où l'on enferme les particularités ethnoculturelles et un public où l'on intervient et on agit sur la base d'une égalité formelle. Ainsi se sont fondues les successives vagues de migrants qui depuis la fin du XIX ${ }^{\text {ème }}$ siècle ont constitué la population française (un tiers des Français actuels au moins ont un grand-parent né à l'étranger). Des études menées actuellement montrent les processus par lesquels est passée cette assimilation (l'école, les organisations politiques et syndicales).

a) par les exigences multiples de la reconnaissance de l'ethnoculturel et du religieux comme composantes légitimes du champ public. La fixation dans le privé de ces éléments producteurs des différences antagoniques à l'égalité formelle est de plus en plus contestée, non seulement par les populations d'origine maghrébine, mais également à travers les tentatives d'organisation apparues parmi les gens d'origines arménienne ou juive, sans parler de certaines résurgences dans les milieux catholiques.

b) par une campagne idéologique menée par l'extrême droite et qui, durant cette décade, a trouvé un écho grandissant dans l'ensemble de la société. Les identités ethnoculturelles sont absolutisées et rattachées à l'extérieur. Ceux qui sont impliqués dans ces identités collectives sont ainsi produits en étranger. Cette frontière est interne à la société, en aucune manière elle n'épouse la nationalité, dans la mesure où une grande partie de ceux qui peuplent la cible possèdent la nationalité française. Cette campagne a pour effet de créer une minorité marginalisée, mais endogène.

Ainsi sont mises en place les conditions de la réalisation du programme dit de la «préférence nationale» (les Français d'abord, dans l'emploi, à l'école, aux services 
sociaux et de santé, au logement, aux prestations familiales, etc ... ). Ces propositions, dont la force démagogique est évidente, détruisent dans la pratique les fondements mêmes de l'action publique.

\section{RÉSUMÉS}

During the last 15 years, French society has been the setting for the development of two crosscutting phenomena : the settlement of an important non-native population (particularly, more than 3 million from Maghreb) and a severe economic and social crisis (massive unemployment and erosion of social protections). The crisis shatters former certitudes and destroys the symbolic devices constructed during some thirty years of exceptional development and modernization. The coïncidence of the two phenomena brings about a rise of xenophobia. The foreigner, being the principal carrier of the social "negative", thus becomes the central reference for natives who attempt to expel poverty and dependency, both of which have become omnipresent while people had imagined they had been eradicated. The paper tries to define this process as it takes place in the everyday life of urban peripheries.

INDEX

Mots-clés : vie quotidienne, identité, étrangers, altérité, France

Keywords : everyday life, identity, strangers, alterity, France

\section{AUTEUR}

\section{GÉRARD ALTHABE}

EHESS, Paris 